\title{
Simulating intertwined design processes that have similar structures: a case study of a small company that creates made-to-order fashion products
}

This is an author-created post-print of the article published in:

International Journal of Product Development, Vol. 14, Nos. 1-4, pp. 118-143. (2011)

DOI: 10.1504/IJPD.2011.042296

David C. Wynn*

Cambridge University Engineering Department, Engineering Design Centre,

Trumpington Street, Cambridge CB1 2RX, UK

E-mail: dcw24@cam.ac.uk

${ }^{\star}$ Corresponding author

\section{Claudia M. Eckert}

Faculty of Maths, Computing and Technology, The Design Group, The Open University, Walton Hall, Milton Keynes MK7 6AA, UK

E-mail: c.m.eckert@open.ac.uk

\section{P. John Clarkson}

Cambridge University Engineering Department, Engineering Design Centre,

Trumpington Street, Cambridge CB1 2RX, UK

E-mail: pjc10@eng.cam.ac.uk

\begin{abstract}
The authors use simulation to analyse the resource-driven dependencies between concurrent processes used to create customised products in a company. Such processes are uncertain and unique according to the design changes required. However, they have similar structures. For simulation, a level of abstraction is chosen such that all possible processes are represented by the same activity network. Differences between processes are determined by the customisations that they implement. The approach is illustrated through application to a small business that creates customised fashion products. We suggest that similar techniques could be applied to study intertwined design processes in more complex domains.
\end{abstract}

Keywords: design customisation; concurrent projects; resource limitation; design process simulation. 


\section{Introduction}

Coordinating the resource needs of overlapping development projects is challenging for many companies. A new project does not usually start when it is convenient for the company, but rather when the customer requests it, when a market opening arises or when it is required by other external drivers - such as legislative change necessitating a new product range. New projects have to be started before others are completed, creating knock-on impact through competition for shared resources. Human resources might need to be maintained, even in periods of lower demand.

Understanding and controlling the flux of projects and resources is critical for many companies. While some companies cannot afford to turn away business, others can - to some extent - select the projects that they take on board. If they understand their workload, to manage risk they might not bid for a project or might refuse a request for a new product, or they might choose to offer longer delivery schedules or higher prices for their products. Obtaining additional resource at peak times may not be possible, may add to the cost of a project or may jeopardise other projects from which those resources must be borrowed. Unrealistic schedules can, therefore, have significant impact on profitability.

These resourcing issues can be particularly problematic for companies that produce customised products, i.e., many different types of product that each require a certain amount of change from a basic design or from a previous version of a similar design. Each request for customised products is a potential small project so that companies which make customised designs have to deal with a multitude of intertwined redesign projects. Ideally, the new products should be easily adapted by making changes to an existing design, through a predictable and perhaps standardised process. Although this situation does exist in some companies with mature products and processes designed with customisation in mind, in other cases, it remains difficult to predict exactly when new orders will arrive or just how much design rework will be required to meet them. These uncertain and dynamic environments require dynamic rebalancing of resources across multiple projects, as companies try to minimise the risk of late delivery.

This paper illustrates how computer simulation of design and production processes can help to understand some of the issues involved in managing the creation of customised products, in particular where the processes are interdependent due to demands on shared resources. Drawing on a case study of a small company producing personalised fashion products, we argue that, even though each batch of products may be different, the customisation processes are very similar when viewed from a certain perspective and level of abstraction. We use this case study to show that a generic process model can be constructed and used to simulate the process of responding to different order types. We also show how the simulation can be used to explore the impact of taking on a new order under different workloads and identifying the loading level at which other projects are likely to be delayed. By illustrating that a simulation model can be constructed, calibrated and used to explain key features of a creative redesign process that appears relatively unstructured at first sight, we argue that a similar approach could be used to study and improve design change processes in complex engineering environments that also involve iteration, uncertainty and responsiveness. The key issues are to identify an appropriate form and level of abstraction for modelling the process, and to model the differences between projects in terms of the changes that initiate them.

The remainder of the paper is organised as follows. In Section 2, we discuss the process management-related challenges of making modifications to a design, arguing that many of the issues can be viewed as resource assignment problems. Section 3 explains the 
principles of a generic simulation model, which can be implemented to explore such problems for a specific company situation. Section 4 introduces the case study which is used in Section 5 to detail the approach. Section 6 illustrates how the model can be used to explore one particular issue revealed by the case study. Section 7 highlights limitations and generally applicable insights, and Section 8 concludes.

\section{Design change and customisation}

Most engineering products are designed by modification from existing ones. The distinction between new design based on a predecessor and customisation of a product for specific customer needs is not well-defined; both can be seen as forms of change process. Change is also a prominent feature of ongoing design processes. Drawing on interviews with engineers and managers from 13 companies in 7 industries, Fricke et al. (2000) estimate that $30 \%$ of all work in these companies arose from some sort of change.

The literature on customisation (e.g., Spring and Dalrymple, 2000) provides insights regarding how to minimise the effort and problems associated with customisation projects. Many of these approaches are design-led. In (mass) customisation, for instance as described by Piller (2007), a company typically offers a number of base designs and option packages, but sometimes also has to engage in design modification. It is very much a business decision where a company positions itself along the spectrum of offering a few simple options vs. offering a fully bespoke service. From the point of view of the customisation process, one of the main differences is that mass customisation typically aims to ease the implementation of a very large number of small changes, while changeled customisation deals with fewer, larger changes. While the case study and analysis in this paper focuses on the latter situation, many of the problems associated with managing intertwined processes can be recognised in all the customisation approaches. Some of the main issues are outlined below.

\subsection{Variability and risk in change processes}

Change and customisation processes are not only ubiquitous but also difficult to manage in part due to variability. The occurrence of change processes is hard to predict, as changes are often driven by the emerging needs of customers. These emerging needs either result in new customisation projects or in changes to projects that are already in progress. The unpredictability of change processes' arrival and content is exacerbated in companies with a large number of different customers. Furthermore, the cost and duration of individual customisation processes are difficult to predict, due in part to change propagation - a phenomenon which has received much attention in the literature.

For instance, empirical studies have highlighted how changes initiated in one area of a design have the tendency to propagate unexpectedly, requiring additional knock-on change to many other aspects of that design (Terwiesch and Loch, 1999; Eckert et al., 2004; Rowell et al., 2009; Giffin et al., 2009). These studies also suggest that, when knock-on change affects many parts, development projects can be severely delayed and hence run over budget. Ariyo et al. (2006) argue that change can also spread through the dependencies between people who implement them and processes in which they operate. For example, if a change request turns up, but the team with core expertise is not available, another team might address the problem in a very different way. This is another factor that makes the prediction of change processes difficult. 
In summary, change and customisation processes are variable in terms of when change orders will arise, what the required change will be and the extent of rework that will be required to implement the change. To manage change effectively in a customisation environment, it is, therefore, important to be able to forecast the likely cost of making each change, such that products can be priced appropriately. After making these commitments, it is also important to manage the impact of variable demand upon shared resources so that individual projects are not delayed. These aspects of change prediction and managing variability are discussed in the following subsections.

\subsection{Tools to estimate the impact of change and the cost of customisation}

Various tools have been developed to predict the impact of change in engineering products: through modelling dependencies between parameters of the design (Ollinger and Stahovich, 2004), by anticipating changes (Cohen et al., 2000) and by modelling the probability of change propagating between subsystems (Clarkson et al., 2004). However, Fricke et al. (2000) argue that a cost-benefit analysis for changes has to include "the entire product development system", including cost, time and resource utilisation. More recently, authors such as Gärtner et al. (2008) and Ahmad et al. (2009) have begun to consider this further and have proposed methods to assess changes by considering propagation both through connections in the product and information dependencies in the redesign process. Most of these approaches consider change as an isolated phenomenon. None of them explicitly considers the effect of the multi-project environment - factors such as resource loading and interdependency between changes. It seems likely that the ultimate cost of implementing a change or meeting a customisation request can be influenced by the current workload, as well as by propagation effects. Furthermore, the cost of customisation is likely to be influenced by structural characteristics of the customisation process and the policies for managing it. In turn, this suggests that a better understanding of dependencies and interactions in the customisation environment might allow more effective management of change and customisation requests.

\subsection{Managing the variability of change and customisation processes}

The discussion in Subsection 2.1 highlights that there are multiple sources of variability associated with design customisation. This variability is transformed through the system of customisation processes and their interactions to create risk - including risk of late delivery, risk of lost profit and risk of delivering products with insufficient quality. These risks can often be mitigated at the cost of reduced performance; for instance, by only accepting projects once others are completed or by quoting a longer lead time when tendering for products, thereby allowing more room for manoeuvre. Appropriate processes and management strategies can, therefore, help maximise process performance within an organisation's taste for risk. Fricke et al. (2000) draw on case studies in 13 companies to suggest five such strategies to manage change in the context of engineering products: Prevention, front-loading, effectiveness, efficiency and learning and reviewing.

Prevention aims to reduce (or eliminate) the number of changes that arise from errors or as a consequence of other changes. In a customisation environment, change is the core business and cannot be avoided. However, it is possible to design a product that meets multiple demands at the same time. For example, by over-designing a particular feature, it can meet different levels of specification. A number of authors argue that many changes 
can be prevented by front-loading, i.e., investing effort up-front (e.g., Lindemann et al., 1998; Loch and Terwiesch, 1999). The cost of changes can be reduced through Design For Changeability (Fricke et al., 2000; Fricke and Schulz, 2005), which advocates moving away from a design that is highly optimised to a single set of requirements and through "Design for Variety" (Martin and Ishii, 2002), which builds in redundancy for anticipated changes. By using these methodologies, a design team can create a product that will require less redesign effort for future versions. Effectiveness emphasises undertaking effective cost/benefit analysis for each proposed change. Not all engineering changes are immediate or mandatory; some change requests would be best avoided altogether if their cost and benefits were known. Efficiency means that essential changes should be implemented as efficiently as possible by making the best use of limited resources. This is especially relevant in a multi-project environment, where customisation processes cannot always draw on all the people they would like at any time. The final strategy discussed by Fricke et al. (2000) is Learning and reviewing, in which they emphasise that each change offers a chance to improve the design and change processes, as well as the design itself. Thus, future changes can be made easier.

Resource allocation is one of the central issues in implementing such strategies for managing change process variability. For instance, it is usually necessary to consider questions that include: How long will a given change process take and are the resources available to complete it? How will it impact upon other, interdependent processes that require the same resource? How much resource should be dedicated to develop the product offering or process capability, further? The simulation model developed in forthcoming sections provides a way to explore such questions.

\section{Exploring resourcing of customisation processes through simulation}

Simulation experiments are based on a simplified model of reality that allows research questions to be explored and hypotheses to be tested in a virtual environment (Sharif Ullah and Tamaki, 2010). Discrete-event Monte-Carlo simulation is especially appropriate to study the impact of variability, such as variability in arrival time and in the duration of individual activities, upon the whole-system performance (Pidd, 2004).

Simulation is a useful tool to explore problems surrounding resourcing of customisation processes because these problems are difficult to examine empirically due to the challenges of isolating the important influencing factors, of obtaining access and of obtaining sufficient data. Applying simulation to explore design process problems is wellestablished in the literature (Browning and Ramasesh, 2007). However, most authors in this community focus on simulating design processes in isolation and do not directly consider inter-project resourcing problems while simulating change processes. Others, such as Loch and Terwiesch (1999), do analyse capacity issues associated with intertwined change processes, but do not model the differences between those processes. We argue that capturing the differences between design customisation processes is important because they are each to some extent individual and thus can have different resourcing demands. The model presented here aims to provide a platform to explore the issues associated with multiple, intertwined customisation processes, while also recognising that those processes can differ significantly in their details. 


\subsection{Overview of the approach}

Our simulation model assumes that many customisation environments can be modelled using the same basic framework, which places the design customisation process into the context of a system of intertwined processes executed to create customised products.

The generic model is shown in Figure 1. In overview, it assumes that when an order for customised products is accepted, a commitment is made regarding when the products will be delivered. Design and production processes whose contents depend on the specifics of the order are then executed. When this is complete, our model assumes that the company may face penalties if the promised delivery date has not been met. (For simplicity, these penalties are not modelled directly but are assumed to be related to the magnitude of delays.) The model further assumes that, given the need to work on multiple projects concurrently, a company will dynamically rebalance the available resources in an attempt to minimise the total delay.

Each aspect of the generic model is described in greater detail below.

Figure 1 Generic model of the process of responding to an order for customised products

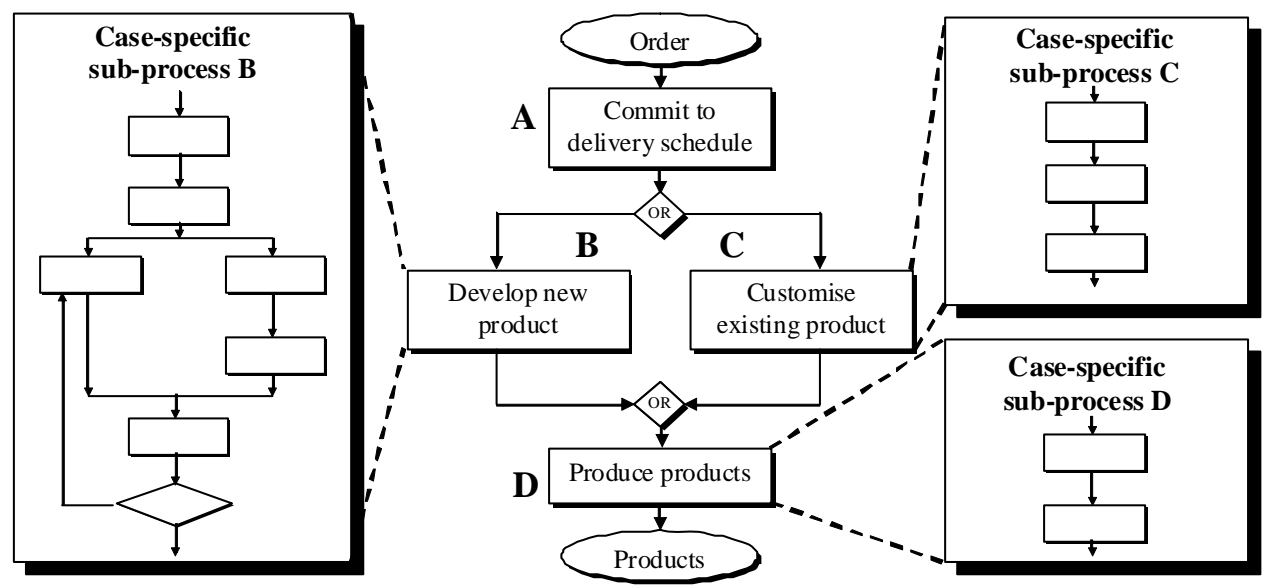

\subsection{Simulating a single process for creating customised products}

As described above, the structure of the process for creating customised products is assumed to be common across projects and companies. When an order is received, an estimation process is followed to create the time commitment that will be made for its delivery (subprocess A). Depending on the degree of customisation required by the order, either a full (re)design process is carried out (subprocess B) or, for simpler customisations, a generic change process is undertaken (subprocess $\mathrm{C}$ ). Finally, one or more customised products are realised (subprocess D) and returned to the customer. The tasks, information flows and resource requirements comprising each of the four case-specific subprocesses (A, B, C and D) are assumed to depend on the particulars of the customisation process in a given company and for a given type of product. 
In the case study described later in this paper, each of the four case-specific subprocesses was modelled using the Applied Signposting Model (ASM) (Wynn et al., 2006). The ASM is a discrete-event simulation modelling tool based on graphical depictions of workflows; it was chosen due to the authors' familiarity with the notation, although other discrete-event simulation approaches could equally have been used. In overview, each subprocess was modelled according to the following scheme:

- $\quad$ The subprocess begins with an input, for instance, the need for customisation. Receipt of this input allows the first task in the subprocess to be attempted.

- The duration of each task within the subprocess is modelled as a Probability Density Function (PDF), which allows the variability associated with (re)design processes to be incorporated into simulations.

- When completed, each task generates new information allowing the successor task(s) to be attempted. Where a task has multiple predecessors, all predecessors must be completed prior to beginning that task.

- All tasks require resources. For instance, executing a certain task may require a designer with certain skills for the entire duration of that task. These resources are assumed to be shared across all tasks, subprocesses and customisation projects.

- Certain tasks in a subprocess, for instance, those representing design evaluation activities, may have more than one outcome. We assume that the probability of each outcome occurring can be estimated. Each outcome may lead to a different successor task. For instance, the model might be organised such that if iteration were required to refine the design or correct errors, a certain set of design tasks should be revisited. In this case, following iteration of all these tasks, the evaluation activity would be encountered again and the procedure repeated. If iteration was not required, the next downstream task would become available.

As discussed in Section 2, in reality, all orders for customised products differ - generating projects that may require different balances of work and which may incur different risks. Our model assumes that, while the structure of activities and information flows within each subprocess remains constant for all change processes that could occur in the modelled situation, the behaviour of tasks within the four case-specific subprocesses is contingent on the details of each order. These details may be modelled as parameters associated with the order. For instance:

1. Does the order require significant redesign or can it be met through more straightforward customisation of an existing design?

Some customisation orders are relatively simple or can be easily implemented given the product characteristics. Eckert et al. (2004) identified that these types of engineering change are typically carried out through a canonical process involving a similar series of steps - regardless of the cause of the change. For instance, they describe a diesel engine company that follows a standard process for assessing change requests: a request typically comes to an engineer who helps the company to identify an existing engine that would meet their needs. They then raise a change request for the required modifications, which must be approved and then carried out. The engines 
are then slotted into the production schedule. However, in other cases, a customisation process might be sufficiently complex that it can be viewed as a (re)design process in its own right.

To account for these very different types of customisation process, we use an approach similar to Adler et al. (1995) by assuming that orders can be categorised into two types: those which require a full (re)design process and those which can be met through a simpler generic change process such as that outlined above. This governs the choice between subprocesses B and C in Figure 1.

\section{How many production units are required?}

\section{Do other order details influence process behaviour?}

Depending on the specific situation being modelled, it may be appropriate to further specify the behaviour of the case-specific subprocesses in terms of additional parameters that describe the order. Depending on the case at hand, this could be approached using one of several methods. For instance, Bashir and Thomson (1999) discuss a parametric model of complex design, in which the duration of design work is shown to be related to the complexity of the design problem at hand. Similarly, in software engineering, function point counting is commonly used to assess the time required to implement given feature sets. Ahmad et al. (2009) argue that an engineering redesign process is often a subset of the original design process for that product and that the redesign activities can be identified by tracing the changed requirements to the design tasks that first implemented them.

In summary, the model shown in Figure 1, when detailed with case-specific subprocesses, forms the basis of a Monte-Carlo simulation of the process for responding to a request for customised products. The cost and duration of the simulated process are governed by the values of the case-specific order parameters and by the availability of resource to perform tasks in the workflows.

\subsection{Simulating multiple, concurrent customisation processes}

The model described in Subsection 3.1 is used as the basic building block from which multi-project simulations are constructed:

- It is assumed that orders for customised products arrive at intervals. The times between arrival of orders and the values of the case-specific parameters that describe each order are described by probability distributions. These distributions must either be estimated for the situation being modelled or may preferably be based on historical data. This is illustrated through the case study in forthcoming sections.

- $\quad$ Each order is met through a new instance of the process described in Subsection 3.1. During simulation, multiple overlapping instances of the process shown in Figure 1 are typically in execution at any given time. Each instance of the customisation process is influenced by the parameters of the order that generated it, the availability of resource and the variability in task duration and outcome, which reflects the uncertainty inherent to design and change processes. 
- When multiple projects execute concurrently, they are interdependent - because the tasks within each customisation process compete for attention with other tasks that require the same resource across all the projects in execution (Figure 2). Our model balances resources dynamically by assigning them to the highest-priority project at any given time. The priority of each project is assumed to be related to the actual progress to date, specifically whether actual progress is less than expected given the date at which delivery was promised. This means that, as a project falls behind, resource is borrowed from other projects that are less urgent to expedite its completion. This dynamic balancing reflects the fire-fighting behaviour that we have observed in many companies (Eckert and Clarkson, 2010).

Figure 2 Multiple overlapping instances of the customisation process are in execution at any time

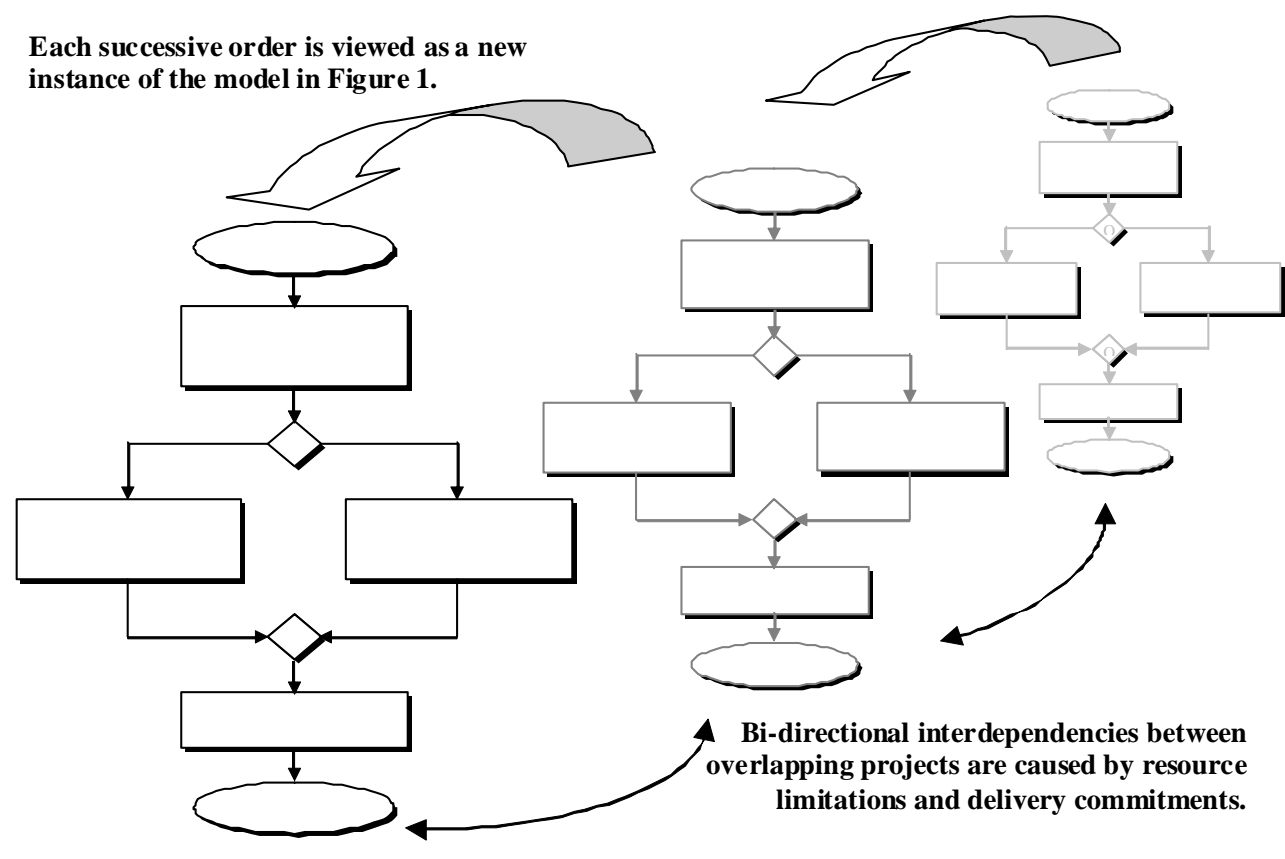

The logic of the intertwined process simulation is illustrated in Figure 3, from the point of view of an individual project. After committing to deliver at a certain date, the first task requiring attention is identified. The priority of the task is then calculated as explained below. The project must wait until resource becomes available and the priority of the next pending task is greater than that of all other pending tasks waiting for the same resource, across all projects. While a project is waiting, the priority of the pending task is continuously recalculated. When the resource is assigned, that task is executed. After completion, the next task is identified and the logic repeats until all tasks in the project are finished. 
Figure 3 Overview of the simulation logic

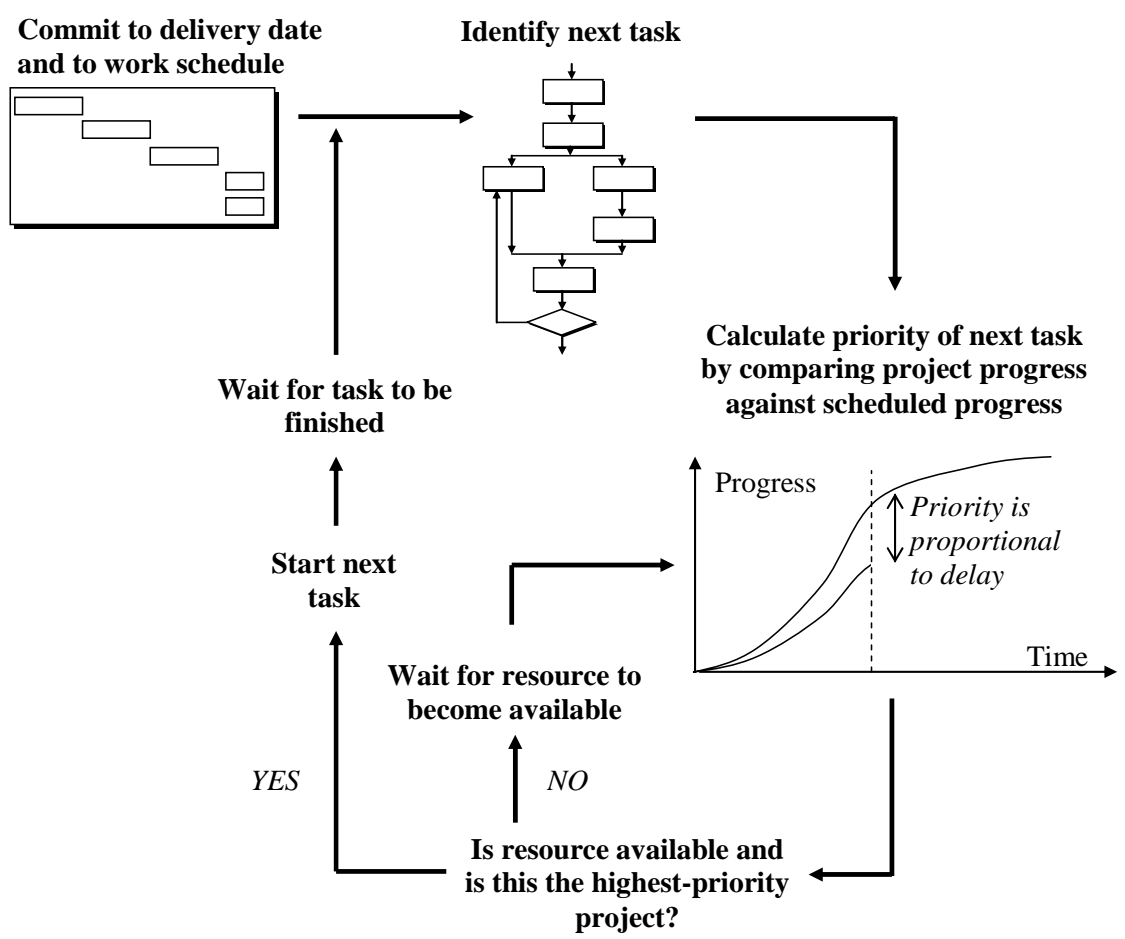

The priority of a task $i$ in the project $j$ is calculated according to equation (1):

$$
P_{i j}=\frac{\left(t_{(\text {elapsed })}-t_{j(\text { start })}\right)}{\left(t_{j \text { (required })}-t_{j(\text { start })}\right) \times t_{i(\text { expected })}}
$$

where

- $t$ (elapsed): The simulation time elapsed since the start of the simulation.

- $\quad t j$ (start): The simulation time at which the $j$ th order was received.

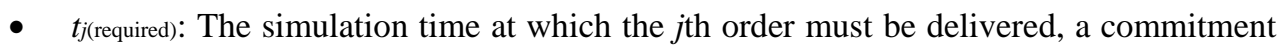
made when the $j$ th order was received.

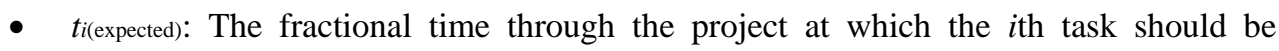
completed.

Equation (1) is arranged such that the priority of the next pending task in every executing project will increase over time until that task is executed. When the task is completed, the completeness of the project will increase and thus the next pending task will have lower priority. Hence, the resource may be allocated to another project that has fallen behind in the meantime. 


\subsection{Summary}

The generic simulation model presented in this section is intended to incorporate key issues observed in practise and reported in the literature: namely, the dependency of the (re)design process upon the work that needs to be done; the variability of the design process; and the need to balance resources across intertwined projects to reduce the risk of late delivery. The model is intended to provide a high-level framework that is generic across many customisation contexts. It thus requires implementation to describe, simulate and explore problems in a specific company. In the following section, we use a case study of a real customisation environment to illustrate how the model can be implemented and to show that it can realistically simulate real-life processes.

Considering Figure 1, many of the strategies to manage change process variability that were discussed in Subsection 2.3 are related to the subprocess entitled 'Commit to schedule'. For instance, committing to an unrealistic schedule when a new order is received is likely to increase risk of delivering late projects, since the new order will place stress on the entire system of intertwined processes - potentially delaying many orders. On the other hand, if it is recognised that the process is close to capacity, the next new order could be assigned a longer duration or rejected altogether. Consideration should also be taken of the change management approaches such as front-loading that were described in Section 2; if too many orders are accepted, then key personnel might be too busy to contribute to these activities, yet if not enough are accepted, the company may not make enough money.

There is thus a need to understand the impact of making new order commitments upon the ability to deliver other projects on time and on the company's ability to exploit opportunities for improving the process. This impact is likely to depend upon the variability of the process, in terms of order arrival, iterations etc., as well as the interactions between projects caused by dependency on shared resources. Some of these issues are explored in Sections 4-6 through a case study.

\section{Case study}

This section describes a case study of a small business that produces bespoke bags. The business is led by a designer-maker who adapts his stock range of designs to meet small orders, occasionally also taking commissions for new designs for individual customers or bigger companies. The case situation is described in detail below prior to introducing the simulation model that was constructed to analyse it.

\subsection{Methods}

The authors conducted three interviews with the designer-maker, as well as numerous informal conversations. These were undertaken as part of the 'considerate design' research project (Black, 2009) in which he participated, which investigated personalisation and its economic viability as a means to increase sustainability in textiles.

The first interview was recorded and transcribed. It provided background information about the designer's creative processes and the constraints he is facing in producing sustainable designs and as a small business producing locally. The second interview took over three hours; we elicited his design customisation and production process, which is followed for every bag, including his estimated durations and failure risks for individual 
tasks. We made notes, took the pictures used in this section and developed a process map interactively with the designer. Finally, a third telephone interview was used to gather numerical data, which was used to calibrate and verify the simulation.

\subsection{Background to the case study}

Steven Harkin is an acclaimed UK accessories designer who has widely exhibited his work through galleries world-wide. He runs his own business designing and making a range of high-quality bags. At the time of writing, he works with a full-time assistant.

Harkin offers a range of basic designs, which are produced to order in small batches by him and his assistant. When placing an order, the customer selects a colour combination from a range of organic leathers in different colours. While most orders are standard shapes and sizes, some are customised to individual measurements and to meet other requests, such as custom fittings. Examples of bags customised from two ranges are shown in Figure 4.

Figure 4 Examples of personalised bags by Steven Harkin, based on two different basic designs
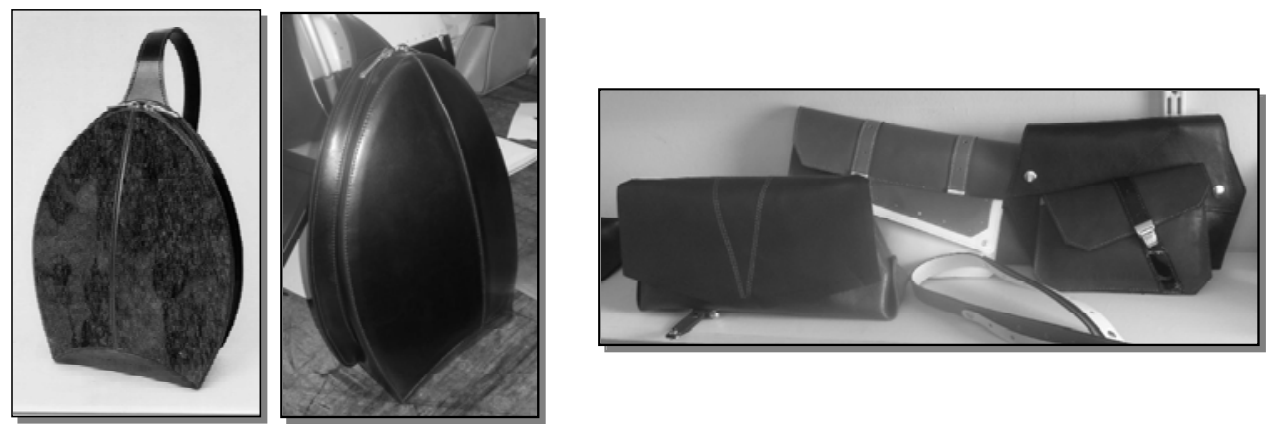

Harkin sells a significant fraction of his bags in trade fairs, where the buyers of independent shops purchase comparatively small orders. His orders come in batches and, as with engineering customisation, with each order he needs to give a realistic estimate of how long delivery will take. While many of his ideas are derived from existing bags in his range, Harkin responds to evolving fashion trends by developing new ranges each season. His business is, therefore, a mixture of customisation and new design, in which design and production are closely linked.

\subsection{Overview of the design process}

Harkin starts the development of a new bag by making sketches such as that shown in Figure 5. The bags must be assembled from several pieces of leather, so he makes paper cutting patterns for the main parts before making partial physical prototypes. This ensures that the $2 \mathrm{D}$ patterns assemble into the right $3 \mathrm{D}$ shapes, that the seams can be worked through the joining machines, that they are not unsightly and so on. Having great experience in designing and making bags, Harkin can switch smoothly between sketches, 2D cutting and a 3D prototype. Nevertheless, a physical prototype using the right materials is necessary to ensure that the components can be reliably assembled. There are often several rounds of iteration involving experimentation with new materials and assembly 
techniques. Once the details are right, the fixtures and fittings must be developed, which in turn can lead to changes in the details. This can be time-consuming as the details can be quite intricate for some designs. The process can be highly iterative as Harkin must source appropriate fittings, develop new assembly techniques and consider durability while maintaining pleasing aesthetics.

Figure 5 The model of Harkin's bag design process (subprocess B in Figure 1), showing different stages of prototype development

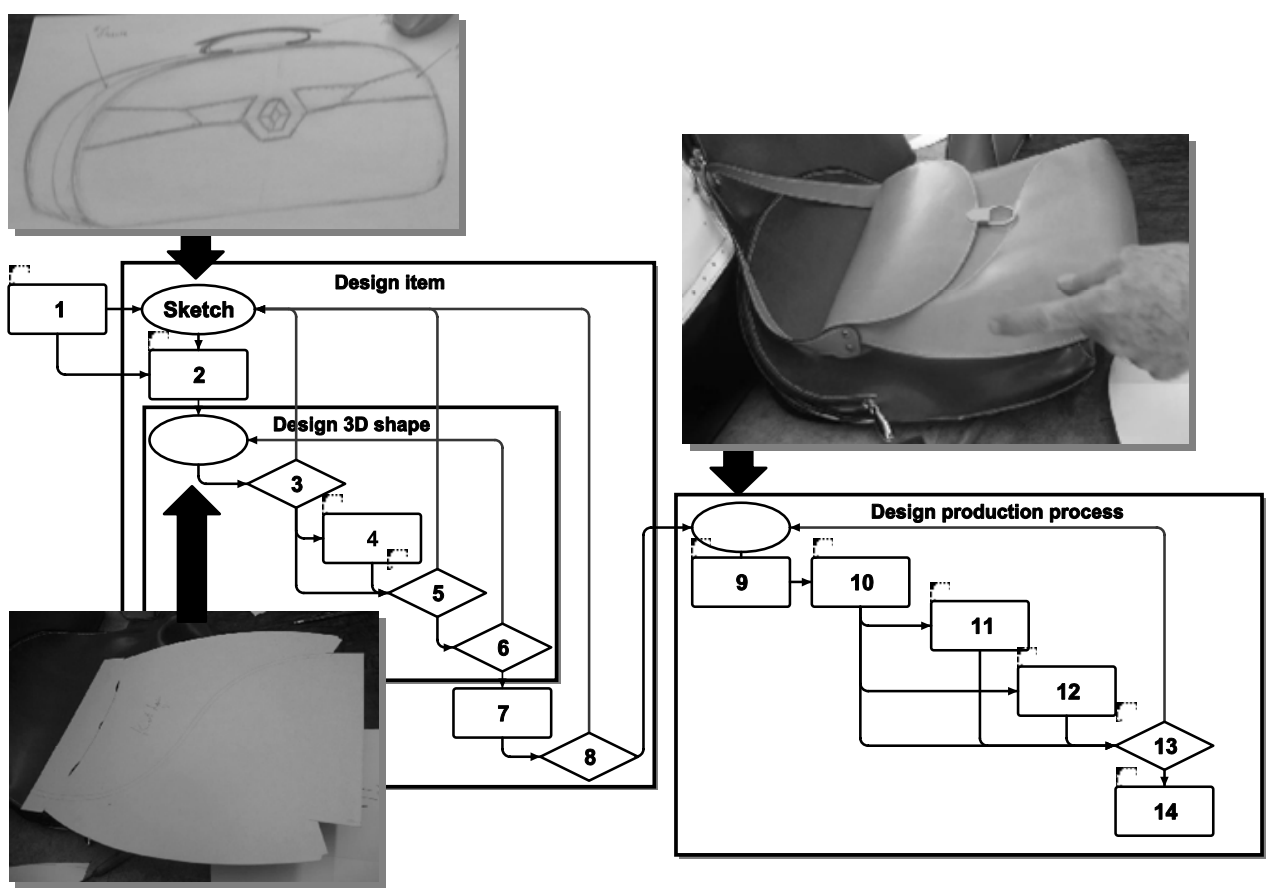

Finally, one or more complete prototypes are made to work out any final problems with the production process and to finalise the sequence of operations required. Samples may then be made in several variants, to be taken to trade shows to generate orders.

While drawing on experience of similar designs, the process still involves significant overhead and variability arising from unpredictable design iterations. Customising existing designs is usually far more straightforward. In cases where the 3D shape is similar to an existing product and the material is in stock, the cost of customisation is very similar to producing a copy of an existing variant - because each item is hand-made anyway. However, differences in shape or fittings can sometimes require different cutting patterns, increase the scope for mistakes and reduce economies of scale.

Some of Harkin's ranges are more successful than others. While it is to some degree difficult to predict whether a design will be successful, the most profitable ranges are often those that Harkin worked on for long periods of time to get the details exactly right. He thus believes there is ultimately benefit in spending more time in iterations to refine a design. However, Harkin and his assistant must already work long hours to meet orders; as a small business taking time away from the income-generating production process to refine details can be difficult to justify. 


\subsection{Resource-related challenges}

Like many small business owners, Harkin is extremely committed to his work, and often works very long hours. He reported spending roughly 300 working days per year designing and making bags, plus around 30 days attending trade fairs to generate orders. His assistant spends 180 working days per year on order processing. Of this time, about $80 \%$ of the total is spent producing bags to an existing design and $12.5 \%$ on designing new bags and ranges. The remainder of their time is spent on the administrative tasks related to running a small business.

Harkin's main resource is, therefore, his and his assistant's labour. While they are sometimes able to outsource certain activities to other people, this requires setting things up and is often not a viable option to deal with short-term fluctuations in demand. One of the main approaches Harkin and his assistant can take to manage their workload is to decide whether or not to take a new order and to decide when to commit to its delivery. They do this by making a case-by-case judgement based on their workload, which they monitor using a visual system of post-it notes, which captures the progress of all their orders at any given time. They also try to reduce production effort by collecting small batches of items that require similar operations, e.g., gluing.

Designers like Steven Harkin often find it difficult to estimate the time it takes them to prepare and produce new designs. If they underestimate the time it takes to meet a new order, they make very little profit, can jeopardise the timely delivery of other commitments or can even risk the loss of repeat business. (In reality, the pricing of Harkin's product is not only determined by the design and production costs but also influenced by comparable products in the market.) This is a big problem as Harkin's business comprises a mix of regular orders combined with occasional large orders generated when he exhibits his work at trade shows. Up to 10 of these large orders (comprising 10-50 bags) are received per annum.

\section{Creation and verification of a specific simulation model}

This section explains how the framework introduced in Section 3 was used to create a simulation model of the intertwined customisation processes in Harkin's business. All numeric data for task durations and outcome probabilities were elicited directly from Harkin during the interviews. By comparison against other information given by Harkin regarding throughput and the average time to process each order, it was possible to verify the generic simulation model by showing how it can provide a plausible representation of a real-life situation. The discussion below is organised into one subsection, which explains how the details of Harkin's orders were modelled, followed by four subsections, which respectively explain the construction of each of the four case-specific subprocess shown in Figure 1.

\subsection{Modelling orders, their arrival and their variability}

On average, two orders are received by Harkin in a normal business week. Each order usually comprises 10 items, with a minimum of 5 items (e.g., for a re-order) and a very rare maximum of 50 items. The orders can be divided into two main types, according to the degree of customisation required: 
- Orders requiring major customisation. An order may require fully-customised bags to be designed and then several items produced. To design a fully-customised bag is expected to take around three days. However, as explained above, this process can take less time, or more if problems are encountered, many iterations are required, or time is spent on the details.

- Orders requiring minor customisation. Bags can also be ordered from an existing range, usually with minor customisations requested. These minor customisations can usually be absorbed into the production process because all items must be hand-made anyway.

Orders can be further subdivided according to the complexity of the bag that is ordered. More complex items require a more time-consuming design process, potentially with more iterations, than simpler items. They also require a more time-consuming production process. To model this distinction, we assume orders can be classified into two types:

- $\quad$ Orders for 'complex’ bags, such as the backpacks in Figure 4 (left).

- $\quad$ Orders for 'simple’ bags, such as the grab-bags in Figure 4 (right).

To summarise, in the case-specific simulation of Harkin's process, each incoming order is parameterised in terms of:

- $\quad$ the number of items required.

- $\quad$ the need (or not) for a full design process.

- the complex vs. simple nature of the design.

After the order is received, a scheduled delivery date will also be set.

\subsection{Modelling the 'Develop new product' subprocess (B)}

To detail the subprocess entitled 'Develop new product' (B) in Figure 1, Harkin's bag design process was modelled during the second interview described in Subsection 4.1. During this interview, the process was discussed in detail and a model was interactively refined and verified by discussion with Harkin. The resulting model is shown in Figure 5. It comprises 14 tasks, each between $30 \mathrm{~min}$ and 1 day in duration. Certain tasks may generate information that is fed back to earlier in the process, thus requiring rework of a block of one or more intermediate activities and any nested iteration loops. These reworkgenerating tasks are shown as diamonds in Figure 5.

During the interview, the nominal duration of each of the 14 tasks shown in Figure 5 was elicited from Harkin. He was asked to estimate one or more of the minimum, expected and maximum duration of each task. This provided the parameters for either a triangular PDF, a uniform PDF, or an exact value defining duration, depending on the task at hand. Probabilities of rework were also elicited for tasks that could drive iteration. When estimating all these values, Harkin was asked to consider the process as if it were carried out in isolation, i.e., assuming that no other work was ongoing. He was also asked to focus 
on the process of designing a 'complex' bag, such as the backpack shown in Figure 4. All the values that were elicited are tabulated in Table 1. According to Harkin, the duration of each task undertaken to create a 'simple' design is about $30 \%$ of that required for a 'complex' design. Likewise, he indicated that the probability of any given task causing iterations when creating a 'simple' design would be about $30 \%$ of the same value estimated for when a 'complex' design was being created. We thus multiplied the values in Table 1 by 0.3 to obtain the process for creating a 'simple' bag.

Table 1 Detail of the bag design process, showing data for numbered tasks in Figure 5

\begin{tabular}{|c|c|c|c|c|c|c|}
\hline & \# & Task name & Predecessors & Duration & $t_{i(\text { expected })}$ & $\begin{array}{r}\text { Iteration likelihood } \\
\text { / tasks revisited }\end{array}$ \\
\hline \multirow{8}{*}{ 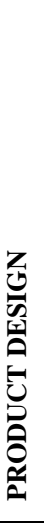 } & 1 & $\begin{array}{l}\text { Create thumbnail sketches } \\
\text { of design concept }\end{array}$ & N/A & $30 \mathrm{~s} / 4 \mathrm{hr}$ & 0.013362 & \\
\hline & 2 & $\begin{array}{l}\text { Make 2D outline of core } \\
\text { aesthetics }\end{array}$ & 1 & $\begin{array}{r}10 \mathrm{~m} / 45 \mathrm{~m} \\
/ 4 \mathrm{hr}\end{array}$ & 0.144837 & \\
\hline & 3 & $\begin{array}{l}\text { Make cutting pattern for } \\
\text { 3D shape, inc. gusset etc. }\end{array}$ & 2 & $90 m$ & 0.181738 & $0.2 / 2-3$ \\
\hline & 4 & $\begin{array}{l}\text { Make patterns etc. for } \\
\text { subsidiary parts }\end{array}$ & 3 & $1 \mathrm{hr} / 2 \mathrm{hr}$ & 0.191783 & \\
\hline & 5 & $\begin{array}{l}\text { Cut parts from similar } \\
\text { material }\end{array}$ & 3,4 & $\begin{array}{r}30 m 70 m \\
90 m \\
\end{array}$ & 0.198908 & $0.2 / 2-5$ \\
\hline & 6 & $\begin{array}{l}\text { Make } 2 \text { or } 3 \text { partial } \\
\text { prototypes to refine ideas }\end{array}$ & 5 & $2 h r / 8 h r$ & 0.36053 & $0.2 / 3-6$ \\
\hline & 7 & $\begin{array}{l}\text { Generate ideas for lining } \\
\text { etc. }\end{array}$ & 6 & $\begin{array}{r}30 \mathrm{~m} 70 \mathrm{~m} \\
90 \mathrm{~m} \\
\end{array}$ & 0.367652 & \\
\hline & 8 & $\begin{array}{l}\text { Assemble prototypes } \\
\text { (average 3) }\end{array}$ & 5,7 & $4 h r 10 h r$ & 0.415159 & $0.2 / 2-8$ \\
\hline \multirow{6}{*}{ 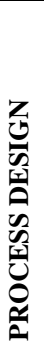 } & 9 & $\begin{array}{l}\text { Plan how to assemble } \\
\text { production versions }\end{array}$ & 8 & $8 \mathrm{hr}$ & 0.437678 & \\
\hline & 10 & $\begin{array}{l}\text { Make full prototype to } \\
\text { test production sequence }\end{array}$ & 8,9 & $8 \mathrm{hr}$ & 0.451147 & \\
\hline & 11 & $\begin{array}{l}\text { Develop internal } \\
\text { prototype }\end{array}$ & 10 & $8 \mathrm{hr}$ & 0.464615 & \\
\hline & 12 & $\begin{array}{l}\text { Identify how to procure } \\
\text { fittings }\end{array}$ & 10 & $8 \mathrm{hr}$ & 0.478084 & \\
\hline & 13 & $\begin{array}{l}\text { Iterate production design } \\
\text { to refine }\end{array}$ & $10,11,12$ & 0 & 0.478085 & $0.15 / 9-13$ \\
\hline & 14 & Define variants & 13 & $5 \mathrm{hr} 6 \mathrm{hr}$ & 0.515135 & \\
\hline
\end{tabular}

\subsection{Modelling the 'customise existing product' subprocess (C)}

Since all products are hand-made anyway, it is assumed that minor customisations can be absorbed in the production process without requiring any specific design rework. Thus, subprocess $\mathrm{C}$ of Figure 1 contains no tasks in the model of Harkin's process.

\subsection{Modelling the 'produce items' subprocess (D)}

Harkin's production rate is variable. However, this depends mostly on the style of bag being produced rather than production process variability. For the large backpack range shown in Figure 1, only around three items may be made per day. For small items, such as purses, which require less cutting and assembly, up to 20 items can be made in one working day. 
For simplicity, and because there was no need to model variability in production, the entire production process was modelled as a single task entitled 'Make one item', representing the production of one entire bag. Within subprocess $D$, this task is repeated as many times as required to produce enough bags to satisfy the order. The time required for each iteration of the 'Make one item' task was modelled to vary according to the type of bag being produced, as described above. The task competes for the same resources as all the design tasks in subprocess B, since Harkin and his assistant must perform all design and production activities themselves.

\subsection{Modelling the 'commit to delivery date' subprocess (A)}

To detail subprocess A, it was assumed that Harkin always schedules a new order by considering the rate at which an order should progress if it were executed without resource constraints. To implement this within the simulation, the date at which each task is expected to be complete was first calculated by executing 1000 runs of the process model presented in Figure 5. The time at which each task $i \mathrm{w}$ as completed was logged for each simulation run. Since each simulation run represented a different possible outcome of the process, this resulted in 1000 values for each task. The average value of this set was

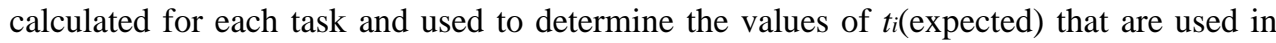
equation (1). These values are plotted in Figure 6 and listed in Table 1.

This is a very simple model of how Harkin schedules his work. It does not take into account, for instance, the impact of current workload on delivery dates or even how the characteristics of each order impacts upon the duration of the customisation process. The verification described in Subsections 5.6 and 5.7 indicates that it nevertheless gives a good approximation of Harkin's process.

Figure 6 Values of $t_{i}$ (expected) indicate fractional time into a project by which each task in Figure 5 is expected to be completed in an 'average' project. Task 15 indicates completion of all production

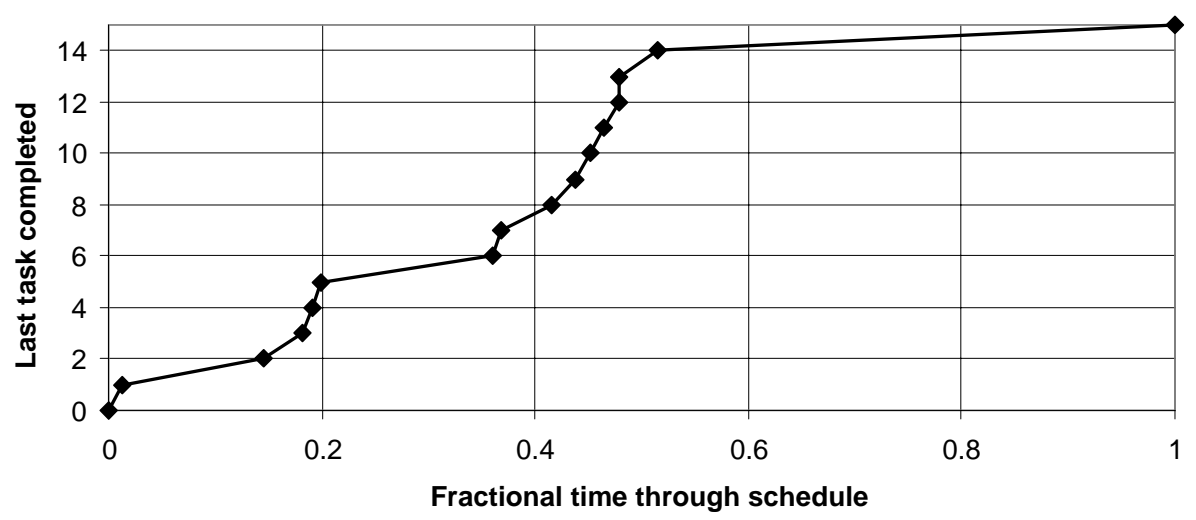

\subsection{Verifying the model of the process in isolation}

The information given above allowed simulation of Harkin's (re)design and production process for different types of order. Verification of the isolated process model was undertaken by comparing the simulated range of durations shown in Figure 7 against 
Harkin's estimate of the duration of the overall process duration, for the two cases of designing a simple bag vs. designing a complex bag. In both cases, production of 10 units was included. This comparison lent credibility to the simulation by showing that it was able to plausibly integrate information that was elicited from several points of view and from several levels of abstraction: estimates of the individual task durations, the model of the activities and information flows and Harkin's estimates of total process duration.

Figure 7 Results from simulating the full (re)design process for a simple bag (left-hand line) vs. the full (re)design process for a complex bag (right-hand line), in both cases, including production of 10 units and assuming one resource is available.

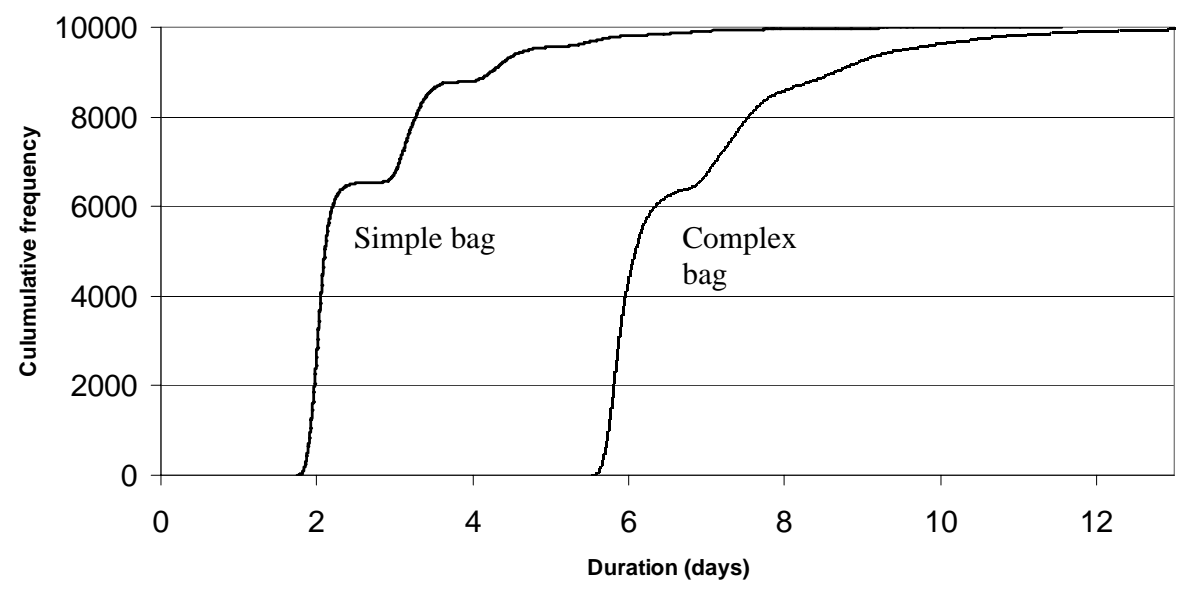

\subsection{Verifying the multi-process simulation}

To verify the multi-process model, seven simulation experiments were run in which the processes of responding to 20 consecutive orders arriving under different conditions were simulated. In all seven experiments, 100 simulations were executed, requiring $20 \mathrm{~min}$ computing time per experiment on a desktop PC. As a starting assumption, it was assumed that all 20 orders were for complex bags, requiring a full redesign process and production of 10 units. Thus, while the experiments accounted for variability in the duration of each redesign process, it was assumed that no variability exists in order arrival. Across each of the seven experiments, the time between each new order was varied systematically to explore the impact of workload upon the time taken to deliver each order. Two units of resource were assumed to be available throughout all the simulation experiments (i.e., Harkin and his assistant). The results are shown in Figure 8, which plots the consecutive orders numbered from 1 to 20 on the $x$-axis against the median time to deliver each of those orders, for each of the seven different experiments. 
Figure 8 Median lead time for processing each of 20 consecutive orders, and variation in median lead times with the time between receiving orders

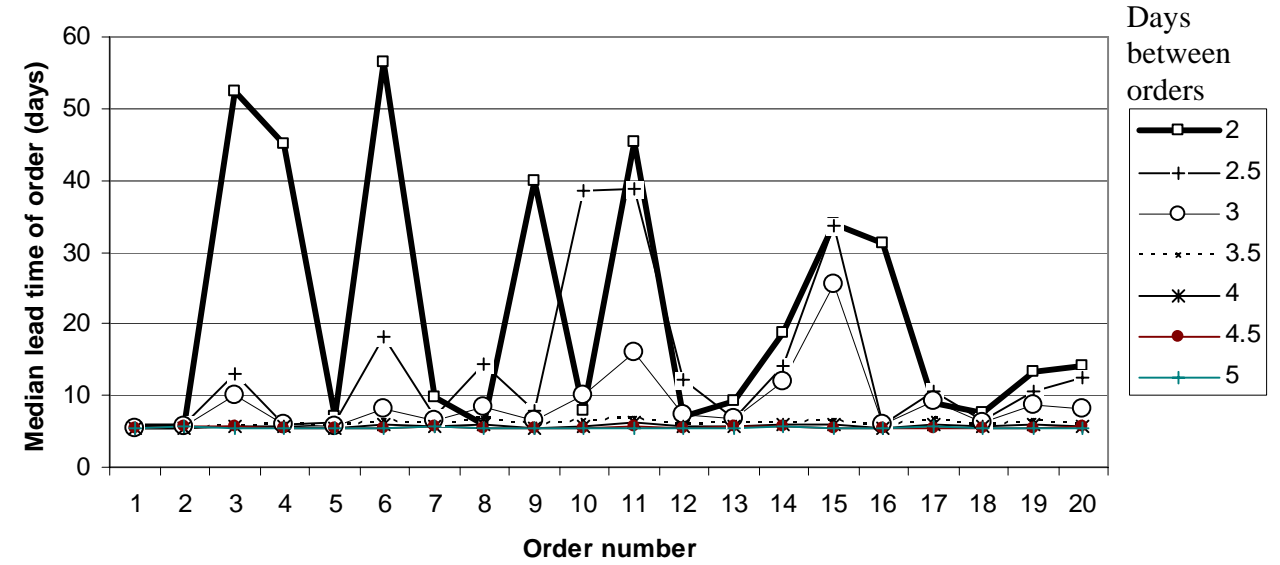

Figure 8 suggests that, when orders arrive at intervals of 3.5 days or greater, they can all be processed with the same median time of about 6 days. This threshold can be easily explained: given two resources, examination of Figure 7 shows that it should be possible to complete each process with a median time of 3 days. However, since the processes are variable, some may take longer than 3 days - thus some headroom is required.

Figure 8 also reveals that instabilities are introduced into the system when the time between orders is reduced to less than 3.5 days. This can be explained as follows. As the time between orders decreases, each process becomes increasingly likely to exceed the time available to it before the next order is received. When this happens, there is a bump in workload, which causes fire fighting; resource is shifted to the delayed project, causing knock-on consequences as the next project is delayed, and so on - impacting several processes downstream, even those which have not yet started. Ultimately, these bumps can be smoothed out by fire fighting. However, the closer the orders become, the less excess capacity exists to enable this smoothing and the more pronounced the delays become.

Assuming two resources, examination of Figure 7 (the intersection of the right-hand curve with the $x$-axis) reveals that the shortest possible average time to complete each process in the simulation is a little over 2.5 days. If orders arrive every 2.5 days or less, even in the best possible case, it will, therefore, never be possible to complete each order before the next arrives - the amount of outstanding work will always increase with time. This explains why the behaviour in Figure 8 becomes so pronounced for low values of time between orders.

These experiments provide some verification of the multi-project simulation by explaining the throughput statistics elicited from Harkin, which are given at the top of Subsection 5.1. In particular, Harkin and his assistant usually process two orders every week: if they accepted orders less frequently, Harkin and his assistant would spend time idle - but in reality, they work very long hours, including most weekends. On the other hand, if they were to accept more than two orders a week, the simulations suggest that they would encounter great difficulty in estimating the time required to deliver any given order. In practise, this is known to be a challenge; Harkin stated that he struggles to determine whether additional orders, such as those generated through trade shows (Subsection 4.4), should be accepted. This issue is explored further in the following section. 


\section{Using the model to explore the impact of accepting new orders}

To illustrate how the multi-project simulation model can be used to help make decisions about resourcing and thus to provide insight into how customisation processes can be managed, this section explores one particular problem: understanding the impact of variation in workload and demand upon the timely delivery of orders. Since Harkin and his assistant usually work close to capacity, as he explained in the interviews and as shown through simulations above, accepting large orders such as those which arise from trade fairs is likely to create some delay to ongoing and downstream projects. For Harkin, it would be useful to understand whether delays caused by accepting a new order are likely to outweigh the profits to be made. Additional simulation experiments were thus undertaken to examine how these delays would vary according to the workload at the time a new order is accepted.

Using the same visualisation as Figure 8, Figure 9 shows what happens when an additional order for 20 units is accepted alongside the sixth order, in the usual case where one order is processed every 3.5 days. This plot reveals that, when Harkin and his assistant are working close to capacity as they usually do, accepting the large order can cause large and unpredictable delays for several months. In reality, they would have to subsequently turn down other orders to regain control over their processes; under these circumstances, the additional order would probably not be worth the trouble.

Figure 9 Impact of accepting an extra order (in parallel with number six) for 20 customised units upon the lead time of downstream orders, for the case of 3.5 days between orders

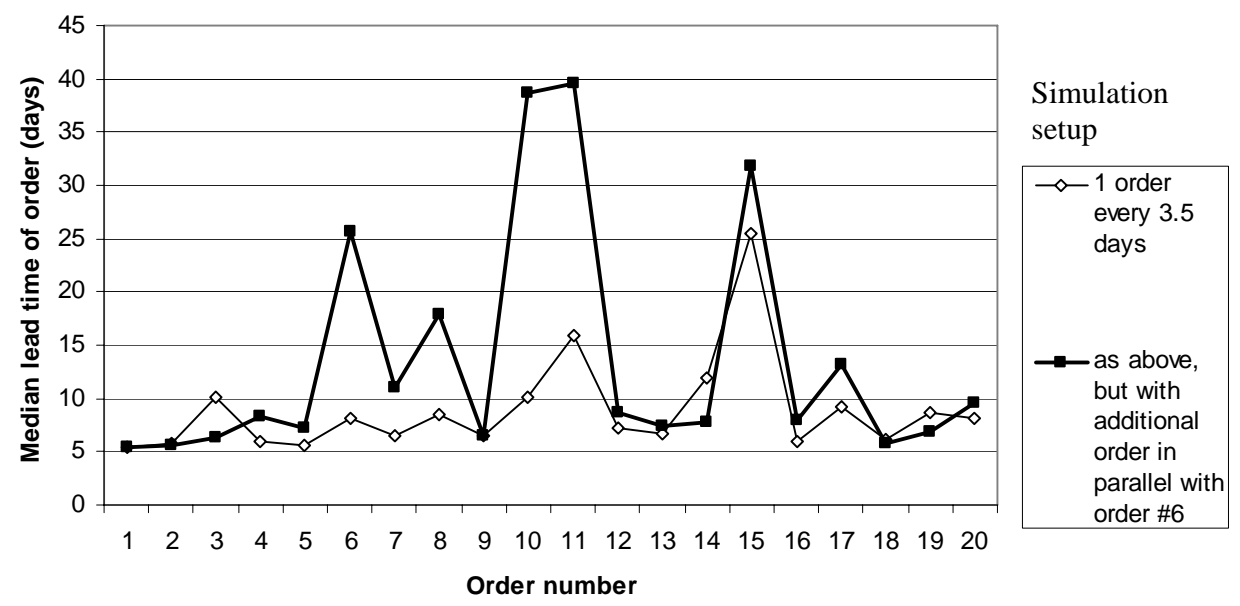

To identify the circumstances under which it would be profitable to accept a new order, additional simulations were run while varying the workload when the order is received. The results, shown in Figure 10, plot workload against the mean delay over 15 orders subsequent to the extra order. This plot suggests that, if Harkin was operating only slightly less than capacity - a new order every 4 days, instead of every 3.5 days - the large orders generated from trade shows could be absorbed with negligible impact. 
Figure 10 Mean delay of the 15 next downstream orders after accepting a new order, and its variation according to the level of workload

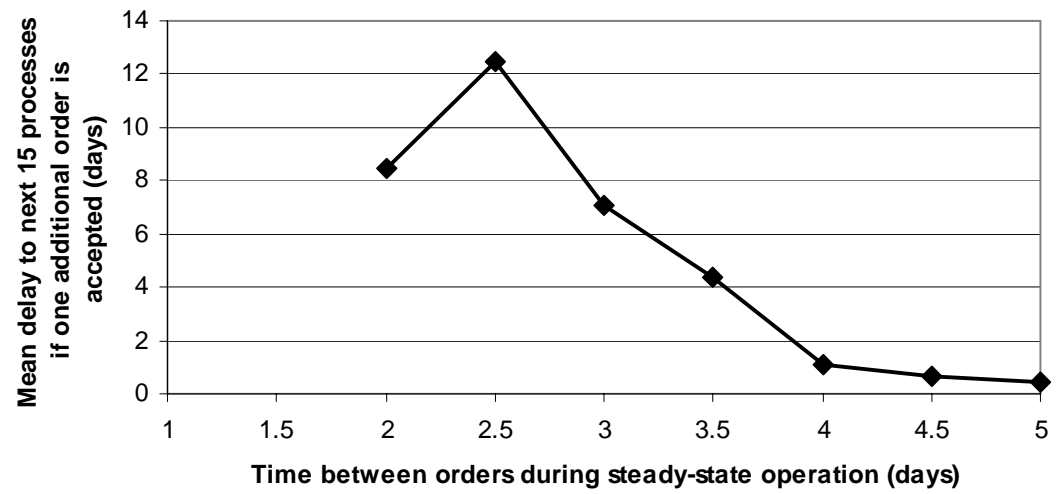

In summary, the simulations in this section corroborate the expected behaviour of Harkin's process - namely, when deciding whether a new order should be accepted, it is important to take into account the level of business when the order is received. Furthermore, Figure 10 provides a way to identify the location of the tipping point where risk of downstream delays becomes unacceptable - about 4 days, in this case (the other tipping point at about 2.5 days occurs when the time between orders becomes less than the minimum time for processing one order; at which point, every project must on average be delayed more than its predecessor).

\section{Discussion}

\subsection{Key contributions}

The main contribution of this paper is to show how a generic structure of design tasks and information flows can be used to simulate the intertwined design customisation processes in a particular company. Our approach recognises that all customisation processes differ and captures the differences between those processes by defining the duration and outcome of each task in terms of the details of the design change which the process must implement. By linking the redesign process to the problem it is intended to address, the approach allows many scenarios to be examined easily, by varying numeric parameters of the simulation. The main challenge in creating such a model is to identify an appropriate form of abstraction when modelling the structure of tasks and information flows.

The case study provides an example of how this approach can be applied to explore questions of resource management in customisation projects. Analysis focused on one particular question which was of interest to the company being studied. However, with minor variations to its configuration, the same model could be applied to explore many other questions related to variability, resourcing and interdependencies between Harkin's design processes. 


\subsection{Validity of the case study to support the findings}

The customisation process system that was modelled in this paper is simple, but real. Although it is clearly far less complex than that for, e.g., aerospace customisation, many familiar issues arise: the need for iteration to explore possibilities and refine designs, the need to test production processes during design and to feedback production problems to alter subsequent versions of the design, and the use of prior designs and experience as the basis to develop new ranges. The relatively simple processes found in the case study made it possible to understand the entire business in reasonable depth and to directly elicit detailed information from which a process model could be built. Since it was unnecessary to make major simplifications to render the analysis tractable, the simulation parameters could be tuned in a realistic way. Finally, it was possible to verify the model by feedback to the process participants and by comparison to throughput estimates.

The case study and the example analysis thus lend support to our approach for modelling intertwined processes by illustrating how models can be created, by showing how the findings from simulation can be explained in terms of case study data, and by showing how simulation can provide plausible guidance for approaching real-life issues. In future, we hope to revisit the company to further verify and test our model. Since the situation which was modelled is relatively simple, it should be possible to obtain relatively high-quality data for simulation by directly observing the entire design and production process. Such observations could further verify the modelling approach, as well as help identify opportunities for improvement.

\subsection{Applicability of the findings to more complex processes}

The simulations presented in this paper were based on the customisation of a relatively simple product. However, while creating the model, we also drew on findings of research on engineering change in collaboration with, amongst others, a diesel engine company (Eckert and Clarkson, 2010) and a helicopter company (Eckert et al., 2004). These studies suggest that engineering change processes can also be modelled as a process generic to a company. Similar findings have been reported by, e.g., Maull et al. (1992), Adler et al. (1995) and Loch and Terwiesch (1999).

Although many features of the environment we studied are similar in type, if not in scope to that of complex engineering, others are different. Some of the key differences include:

- The customisation processes we modelled are largely independent, apart from the need to draw on shared resource. In complex design customisation, change projects may also be interdependent if they modify the same base design.

- In complex design, change processes are not only initiated by external events, such as customer orders, but also by emergent issues within the process and by change to requirements once the project has been started. In engineering, there is also a far more complex structure of tasks and resource requirements within projects.

- In engineering companies, personnel are often more cushioned from the impact of varying workload. This built-in slack may allow peaks in workload to be more easily absorbed. Resource-related issues, such as overheads associated with multi-tasking, differing priorities, and the benefits of 'learning', are also more prominent. 
Many of the differences could be relatively easily incorporated in an extended simulation model developed for a particular case. More generally, we suggest that a key issue in applying our approach to simulate complex design change processes would be to determine how the defining differences between particular change processes could be linked to the details of the redesign problems.

For instance, while engineering change projects typically follow the same formal process of raising a request, identifying possible solutions, assessing the associated risk, selecting a solution and implementing a solution (Maull et al., 1992), this kind of highlevel description would not capture the specific resource requirement, which is required to assess the effects of a given change on the rest of the process system.

The change process would thus need to be modelled in more detail. Identifying a suitable level of detail is likely to require an in-depth understanding of the process, as well as an appreciation of the effort required for modelling and the likely impact of particular assumptions on the simulation results. Furthermore, because the computational effort for simulating intertwined processes is high compared to simulating processes in isolation, the level of detail of the model might also need to be traded-off against simulation time.

\section{Conclusions}

Resource limitations between processes cause those processes to become interdependent. As a result, delays in one project, or the decision to accept new orders, can cause knock-on consequence to other projects in a company, even delaying those which have not started yet. The risks are magnified in semi-structured, dynamic processes, such as design customisation, where additional headroom is required to absorb unexpected delays and iterations. This paper has drawn on a case study of a simple, but realistic situation in a small business to show how the dependencies between iterative, uncertain customisation processes that use shared resources can be studied through simulation. It was shown how to locate the tipping point in behaviour that occurs when workload is high enough that additional orders cause significant disruption.

This type of analysis could provide insight to help companies understand when new orders should be rejected, even if those orders seem individually profitable. Many other questions relating to orders and resourcing policies, such as those mentioned at the end of Subsection 2.3, could equally be explored using the model. However, as with all modelbased approaches, creating a simulation requires that suitable input data be available, including the process structure, as well as estimates of task duration, failure probabilities and arrival statistics. In this case, the model was calibrated by comparing its output to observed throughput of the company.

More generally, it was shown how discrete-event simulation, which is commonly applied to well-structured business and production processes, can also be used to analyse the dependencies between more creative, iterative and responsive customisation processes found in a small business environment. The key to our approach was to identify an appropriate level of abstraction in which the differences between projects are not reflected in the structure of activities and information flows, but only in the properties of tasks located within a generic structure. These tasks' properties can in turn be defined in terms of the detail of the products which the process must deliver. Because the design and production processes studied in this paper display many of the properties of more complex engineering processes - notably including iteration, uncertainty and responsiveness - we suggest a similar approach could be taken to explore the properties of change processes in 
these domains. The model presented here could provide the basis for approaching such an analysis, although many more issues related to the increased complexities of projects and resourcing would also need to be considered.

\section{Acknowledgements}

The case study was carried out as part of Considerate Design for Personalised Fashion funded by the EPSRC/AHRC Design in the 21st century programme. The context of a multi-project environment was analysed as part of the EU Framework 7 CONVERGE project CP-FP 228746-2. We would also like to thank the anonymous reviewers.

\section{References}

Adler, P.S., Mandelbaum, A., Nguyen, V. and Schwerer, E. (1995) 'From project to process management: an empirically-based framework for analyzing product development time', in Schwerer, E.A. and Nguyen, V. (Eds.): Management Science, Vol. 41, No. 3, pp.458-484.

Ahmad, N., Wynn, D.C. and Clarkson, P.J. (2009) 'Estimating the process cost of implementing engineering change alternatives', Proceedings of the 2nd Nordic Conference on Product Lifecycle Management (NordPLM '09), Chalmers University of Technology, Goteborg, Sweden, pp.205-220.

Ariyo, O.O., Eckert, C.M. and Clarkson, P.J. (2006) 'Unpleasant surprises in the design of complex products: Why do changes propagate?’, ASME International Design Engineering Technical Conferences, Philadelphia, USA, DETC2006-99409.

Bashir, H. and Thomson, V. (1999) 'Estimating design complexity', Journal of Engineering Design, Vol. 10, No. 3, pp.247-257.

Black, S. (2009) 'Interrogating fashion: practice process and presentation. New paradigms for fashion design in the 21st century', in Inns, T. (Ed.): Designing for the 21st Century: Interdisciplinary Question and Insights, Gower, pp.299-314.

Browning, T.R. and Ramasesh, R.V. (2007) 'A survey of activity network-based process models for managing product development projects', Production and Operations Management, Vol. 16, No. 2, pp.217-240.

Clarkson, P.J., Simons, C.S. and Eckert, C.M. (2004) 'Predicting change propagation in complex design’, ASME Journal of Mechanical Design, Vol. 126, No. 5, pp.788-797.

Cohen, T., Navathe, S.B. and Fulton, R.E. (2000) 'C-FAR: change favourable representation', Computer Aided Design, Vol. 32, Nos. 5-6, pp.321-338.

Eckert, C.M. and Clarkson, P.J. (2010) 'Planning development processes for complex products', Research in Engineering Design, Vol. 21, No. 3, pp.153-171.

Eckert, C.M., Clarkson, P.J. and Zanker, W. (2004) 'Change and customisation in complex engineering domains', Research in Engineering Design, Vol. 15, No. 1, pp.1-21.

Fricke, E. and Schulz, A.P. (2005) 'Design for Changeability (DfC): principles to enable changes in systems throughout their entire lifecycle', Systems Engineering, Vol. 8, No. 4, pp.342-359.

Fricke, E., Gebhard, B., Negele, H. and Igenbergs, E. (2000) 'Coping with changes: causes, findings, and strategies’, Systems Engineering, Vol. 3, No. 4, pp.169-179.

Gärtner, N., Rohleder, T. and Schlick, C.M. (2008) 'Simulation of product change effects on the duration of development processes based on the DSM', Proceedings of the 10th International DSM Conference, Hanser, pp.199-208.

Giffin, M.L., de Weck, O.L., Buonova, G., Keller, R., Eckert, C.M. and Clarkson, P.J. (2009) 'Change propagation analysis in complex technical systems', Journal of Mechanical Design, Vol. 131, No. 8. 
Lindemann, U., Kleedorfer, R. and Gerst, M. (1998) 'The development department and engineering change management', in Frankenberger, E., Badke-Schaub, P. and Birkhofer, H. (Eds.): Designers: The Key to Successful Product Development, Springer-Verlag, London, pp.169-182.

Loch, C.H. and Terwiesch, C. (1999) 'Accelerating the process of engineering change orders: capacity and congestion effects', Journal of Product Innovation Management, Vol. 16, No. 2, pp.145-160.

Martin, M.V. and Ishii, K. (2002) 'Design for variety: developing standardized and modularized product platform architectures', Research in Engineering Design, Vol. 13, No. 4, pp.213-235.

Maull, R., Hughes, D. and Bennett, J. (1992) 'The role of the bill-of-materials as a CAD/CAPM interface and the key importance of engineering change control', Computing and Control Engineering, Vol. 3, No. 2, pp.63-70.

Ollinger, G.A. and Stahovich, T.F. (2004) 'ReDesignIT - a model-based tool for managing design changes', Journal of Mechanical Design, Vol. 126, No. 2, pp.208-216.

Pidd, M. (2004) Computer Simulation in Management Science, Wiley, Chichester.

Piller, F.T. (2007) 'Observations on the present and future of mass customization', International Journal of Flexible Manufacturing Systems, Vol. 19, No. 4, pp.630-636.

Rowell, W.F., Duffy, A.H.B., Boyle, I.M. and Masson, N. (2009) 'The nature of engineering change in a complex product development cycle', Proceedings of the 7th Annual Conference on Systems Engineering Research (CSER 2009), 20-30 April, Loughborough, UK.

Sharif Ullah, A.M.M. and Tamaki, J. (2010) 'Analysis of Kano-model-based customer needs for product development’, Systems Engineering, issue n/a. DOI: 10.1002/sys.20168.

Spring, M. and Dalrymple, J.F. (2000) 'Product customisation and manufacturing strategy', International Journal of Operations and Production Management, Vol. 20, No. 4, pp.441-467.

Terwiesch, C. and Loch, C.H. (1999) 'Managing the process of engineering change orders: the case of the climate control system in automobile development', Journal of Product Innovation Management, Vol. 16, No. 2, pp.160-172.

Wynn, D.C., Eckert, C.M. and Clarkson, P.J. (2006) 'Applied signposting: a modeling framework to support design process improvement', Proceedings of ASME IDETC/CIE 2006, Philadelphia, Pennsylvania, USA, DETC2006-99402. 